\title{
28 Research Square \\ Identifying predictive patients' characteristics in case of High Flow Nasal Cannula failure when used for post-extubation failure
}

Afra L.E. Terstappen ( $\nabla$ e.terstappen@gmail.com )

VU medisch centrum https://orcid.org/0000-0002-5358-3065

Lenneke E.M. Haas

Diakonessenhuis

Esther S.E. van Seumeren

OLVG

Anja Balzereit

OLVG

Henrik Endeman

Erasmus MC

Research article

Keywords: oxygen inhalation therapy; noninvasive ventilation; reintubation; intensive care; respiratory insufficiency.

Posted Date: November 26th, 2019

DOI: https://doi.org/10.21203/rs.2.17791/v1

License: (c) (i) This work is licensed under a Creative Commons Attribution 4.0 International License. Read Full License 


\section{Abstract}

Background: High Flow Nasal Cannula (HFNC) is a relatively new but broadly used type of oxygen therapy. Hence, not much is known about HFNC in the setting of post-extubation failure. Aim of this study is to identify patients' characteristics that predict failure of HFNC when used for post-extubation failure. Methods: This retrospective, observational study was conducted in a Dutch ICU. Between 2008 and 2014 all subjects aged 18 and older who started with HFNC due to respiratory failure within 2 to 72 hours after extubation were included. Primary outcome was patient characteristics predicting failure of HFNC. Failure was defined as reintubation or death following HFNC. Success was defined as the opportunity to stop HFNC after a non-specified time. Secondary outcome was the difference in length of stay (LOS) and mortality between the success and failure group. Results: A total of 246 subjects were included; in 135 (55\%) cases HFNC failed. The success and failure group were comparable in terms of age, primary diagnosis and duration of mechanical ventilation prior to extubation. HFNC was started $19 \pm$ 15 hours (mean \pm SD) after extubation. Significant differences were found for breathing frequency (success: $22 \pm 7 / \mathrm{min}$ vs. failure: $24 \pm 7 / \mathrm{min}, \mathrm{p}=0.009$ ) and the presence of acute kidney injury ( $51 \% \mathrm{vs}$. $79 \%, p=0.003)$. There was a non-significant difference in $\mathrm{pH}(7.43 \pm 0.07$ vs. $7.40 \pm 0.08, p=0.08)$. After logistic breathing frequency $(p=0.02)$ and $\mathrm{pH}(\mathrm{p}=0.01)$ remained independent predictors of HFNC failure. LOS at the ICU after starting HFNC differed (success: $181 \pm 177$ hours vs. failure: $405 \pm 519$ hours, $p<0.001)$. ICU and hospital mortality were not significantly different between groups. Conclusion: In more than half of the patients HFNC failed when used for post-extubation failure. Breathing frequency and $\mathrm{pH}$ were predictors of HFNC failure.

\section{Background}

High-flow Nasal Cannula (HFNC) is a relatively new therapy and is being used increasingly as primary therapy or as an alternative for non-invasive respiratory support. HFNC delivers a heated and humidified mixture of air and oxygen at high flow rates. HFNC flushes anatomical dead space and improves respiratory efficiency. ${ }^{12}$ In addition it generates positive airway pressure and enables to deliver a more accurate FiOQ. HFNC is known to be used in many different settings, including to prevent intubation in patients with respiratory insufficiency ${ }^{3} 4$ or as an alternative for patients with respiratory failure who have a limitation regarding invasive mechanical ventilation.

Currently, non-invasive ventilation (NIV) plays an important role in the prevention of respiratory failure post-extubation ${ }^{5}$, but recent studies showed an increased mortality if NIV was used in the setting of postextubation respiratory failure. ${ }^{6}$ As a result a new discussion is emerging on the use of HFNC following extubation to prevent respiratory failure. ${ }^{7} 89$ HFNC seems to be a reliable alternative for the prevention of re-intubation in high-risk patient by starting HFNC immediately following extubation. 38910 However, not much is known about HFNC in the setting of post-extubation failure and thus, HFNC is used for postextubation failure without solid scientific evidence about the outcomes. As far as we know, only one small study has been published on this subject. ${ }^{11}$ 
It is important to identify the characteristics that predict success or failure of HFNC and examine the outcomes of HFNC failure. Therefore, the aim of this study is to identify patient's characteristics that predict failure of HFNC in the setting of acute respiratory failure after extubation.

\section{Methods}

This retrospective, observational study was conducted at a 24 beds intensive care unit (ICU) of a Dutch Hospital where OptiFlow ${ }^{\circledR}$ (Fisher \& Paykel Healthcare) was used as a high-flow nasal device. In this ICU the moment of extubation was determined by the attending physician, no spontaneous breathing trials were performed. Between 2008 and 2014 all subjects aged 18 and older who started with HFNC due to respiratory failure within 2 to 72 hours after extubation were included. Subjects who had respiratory failure within 0 to 2 hours after extubation were excluded to guarantee that only subjects with new respiratory problems were included. Other exclusion criteria were any limitation regarding mechanical ventilation (invasive or non-invasive) at the initiation of HFNC or during the study period.

The study was approved by the regional medical ethical committee, a waiver was given for informed consent due to the retrospective and non-invasive nature of this study.

\section{Study design}

Data on subjects' characteristics, length of stay (LOS) and mortality, were obtained from the Patient Data Management System (PDMS). The different variables in the pre-HFNC setting were collected 1 to 4 hours prior to the start of HFNC. Due to the registration method there is no P/F ratio available, but merely a $\mathrm{SpO} / \mathrm{FiO} \otimes$ ratio. Data on reason for admission, primary diagnosis or circumstance in which the respiratory failure occurred, comorbidities and predicted mortality were obtained from the Minimal Data Set (MDS) of the National Intensive Care Evaluation (NICE) in the Netherlands, which is based on the APACHE IV (Acute Physiology And Chronic Health Evaluation) risk model ${ }^{12}$.

Primary outcome of this study were patient characteristics predicting failure of HFNC. Failure was defined as the need of reintubation or death within 72 hours after the start of HFNC treatment. Success was defined as the opportunity to stop HFNC after a non-specified time. Secondary outcome was the difference in LOS and mortality between the success and failure group. The necessity of re-intubation was judged by the attending physician. We took death as part of the definition of HFNC failure as well as secondary outcome. The underlying argument to also make death within 72 hours after the start of HFNC treatment part of the definition of HFNC failure was the fact that otherwise subjects who failed HFNC but were not reintubated and died subsequently, had to be considered, by definition, as HFNC success which is not the case.

Subjects were categorized according to the underlying cause or the circumstance in which the respiratory failure occurred, resembling the different categories in the minimal data set: pneumonia, congestive heart failure (CHF), COPD, post-surgery, other respiratory and other non-respiratory. The other respiratory 
group included subjects with pulmonary embolus, lung cancer, atelectasis and pleural effusion. The other non-respiratory group included subjects with pancreatitis, sickle cell crisis, chest trauma, in the post-CPR setting, gastrointestinal sepsis, acute renal failure, amyotrophic lateral sclerosis and Guillain-Barre Syndrome. All aforementioned diagnosis were not strictly defined, but were found to be present if registered as such in the MDS.

\section{Statistical analysis}

Statistical analyses were performed using SPSS, version 22 Command Syntax References (Chicago, IL, USA). Descriptive statistics were calculated for all variables. The relations between variables and endpoints were tested using Mann-Whitney $U$ tests, independent sample t-tests, Chi-square or one-way ANOVA, depending on the origin and distribution of the date. For all analyses, a $p$-value $p<0.05$ was considered statistically significant. Variables $(p<0.1)$ associated with reintubation after starting HFNC were assessed by means of multivariate logistic-regression analyses with the use of a backwardselection procedure. Variables representing less than $10 \%$ of the population were not used. Post hoc subgroup analyses were conducted for variables associated with re-intubation.

\section{Results}

A total of 246 subjects were eligible for inclusion. Patient characteristics are presented in table 1. Age of this cohort was (mean \pm SD) $69 \pm 11$ years, 147 subjects $(60 \%)$ were male. The main primary reason for admission to the ICU was post-surgery. Acute kidney injury (AKI) and an immunocompromised status were the main comorbidities according to APACHE IV criteria. The in-hospital mortality was lower than the predicted mortality by APACHE IV.

\section{Table 1}


Baseline characteristics of subjects treated with HFNC for acute respiratory failure $<72 \mathrm{~h}$ after extubation

Subjects, $\mathbf{n}$

Male, $n$ (\%)

Age (years), mean (SD)

BMI, mean (SD)

APACHE IV predicted mortality (\%), mean (SD)

Reason for admission to the ICU, $\mathrm{n}(\%)$

$$
\text { Elective surgery/scheduled }
$$

Medical

- Urgent/emergency surgery

Primary diagnosis or circumstances at start HFNC, n (\%)

- Post-surgery

- $\quad$ Congestive heart failure

- $\quad$ Pneumonia (incl. sepsis)

- COPD

- $\quad$ Other, non-respiratory

- Other, respiratory

APACHE IV co-morbidities, n (\%)

- $\quad$ Acute kidney injury

- Immunocompromised

- $\quad$ Chronic renal insufficiency

- $\quad$ Chronic cardiovascular insufficiency

- $\quad$ Chronic respiratory insufficiency

- Hematologic malignancy

Length of stay in ICU (hours), mean (SD)

Mortality in ICU, n (\%)

Table 1. Table of baseline characteristics. BMI=body mass index, APACHE=Acute Physiology and Chronic Health Evaluation score

Table 2 presents the clinical parameters of the total cohort and for both groups (failure and success) before the start of HFNC. In 178 patients an arterial blood gas analysis before start of HFNC was available, 72 patients (40\%) were hypoxemic ( $\mathrm{PaO} 2<60 \mathrm{mmHG}), 28(16 \%)$ hypercapnic $(\mathrm{PaCO} 2>45$ $\mathrm{mmHg})$ and $22(12 \%)$ both.

ble 2 
'arameters before the start of HFNC

ll variables: mean (SD)

$\begin{array}{lll}\text { Total, } \mathrm{n}= & \text { Success, } \mathrm{n}= & \text { Failure } \mathrm{n}= \\ 1346 & \mathrm{p}- \\ 111 & \text { value }\end{array}$

lespiratory parameters

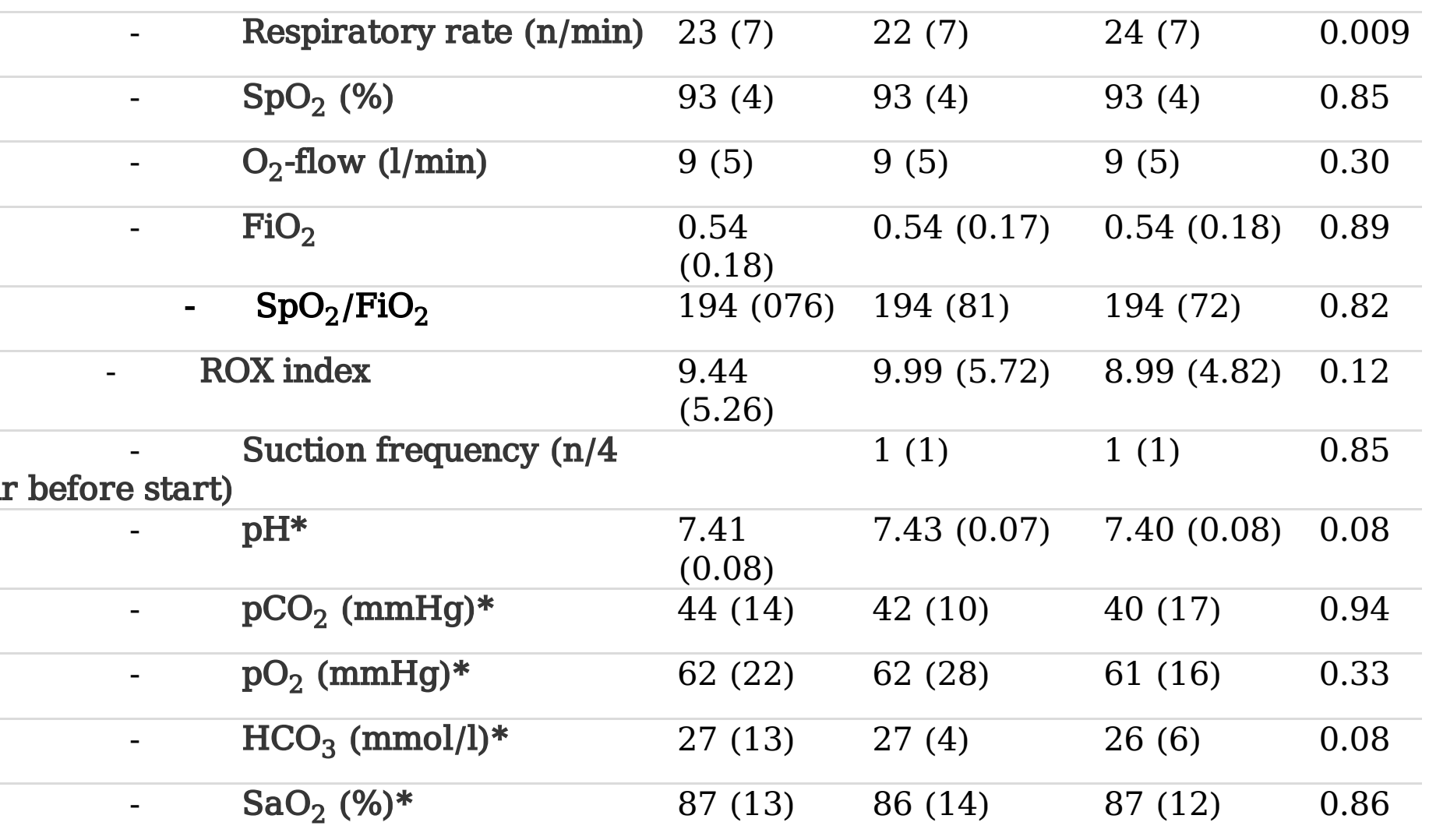

12 therapy before HFNC, $\mathrm{n}$ (\%)

$r$ before start)

$\begin{array}{lllll}- & \text { Oxygen mask/NRBM } & 120(49) & 55(50) & 65(48) \\ - & \text { Nasal cannula } & 95(39) & 40(36) & 55(41) \\ - & \text { Unknown/none } & 31(13) & 16(14) & 15(11)\end{array}$

Iemodynamic parameters

\begin{tabular}{rlllll}
\hline- & Heart rate (b/min) & $91(18)$ & $90(18)$ & $92(18)$ & 0.39 \\
\hline- & MAP (mmHg) & $79(18)$ & $79(18)$ & $79(18)$ & 0.97 \\
\hline tart (ml) & Fluid balance 4 h before & $531(295)$ & $560(309)$ & $507(282)$ & 0.20 \\
\hline
\end{tabular}

$\begin{array}{lllll}\text { lelirium (CAM-ICU positive), } \mathbf{n}(\%) & 99(40) & 46(41) & 53(39) & 0.73\end{array}$

able 2. Table of parameters 1-4 hour prior to the start of HFNC in the success and iilure group.MAP: mean arterial pressure; ROX index: ratio of SpO2/FIO2 to espiratory rate. *Bloodgas analyses were based on samples of 178 subjects; 78 ubjects in the success group, 100 subjects in the failure group. 
In 135 subjects $(54,9 \%)$ HFNC failed; all of these patients were re-intubated. The groups with HFNC success and HFNC failure were comparable in terms of age, sex, primary diagnose or circumstance in which the respiratory failure emerged. There was no difference in HFNC failure between patient who were primary hypoxemic $(49 \%)$, hypercapnic $(54 \%)$ or both $(55 \%)$. The duration of the mechanical ventilation prior to extubation was comparable for both groups (success group: $63 \pm 90$ hours, failure group $110 \pm$ 404 hours, $p=0.43)$.

A significant difference between the groups was found in their breathing frequency prior to the start of HFNC (success: $22 \pm 7 / \mathrm{min}$ vs failure: $24 \pm 7 / \mathrm{min}, \mathrm{p}=0.009$ ). HFNC failure was found to be more present in patients with AKI than patients without AKI (79\% vs. $51 \%, p=0.003)$. No difference was found in the APACHE IV score between the success and failure group.

Arterial blood gas was obtained 1 to 4 hours prior to the start of HFNC. In this arterial blood gas both pH ( $7.43 \pm 0.07$ vs. $7.40 \pm 0.08, p=0.08)$ and $\mathrm{HCO}_{3}(27 \pm 4$ vs. $26 \pm 6, p=0.08)$ showed a tendency to statistical significance.

For the logistic regression analysis a total of 177 subjects were included. After logistic regression breathing frequency $(\mathrm{p}=0.02)$ and $\mathrm{pH}(\mathrm{p}=0.01)$ remained independent predictors of HFNC failure.

There were no differences in IC mortality (success: $7 \%$, failure: $15 \%, p=0.06$ ) and in-hospital mortality (success: $14 \%$, failure: $24 \%, p=0.07$ ) between the groups. Twenty-five patients were lost to follow-up by reason of a transfer to another hospital.

Post-hoc analysis showed a significant higher chances of failure in case of a lowered $\mathrm{HCO}_{3}\left(\mathrm{HCO}_{3}<22\right.$ $\mathrm{mmol} / \mathrm{L}$ vs $\mathrm{HCO}_{3}>=22 \mathrm{mmol} / \mathrm{L} ; \mathrm{OR} 6.33$ (Cl 95\% 1.81-22.22); $\mathrm{p}=0.001$ ) or an increased breathing frequency (breathing frequency >= 30/min vs. breathing frequency <30/min; OR 2.28 (Cl 95\% 1.10-4.71), $p=0.02)$. In case of a breathing frequency $>=30$ HFNC failure was $29 / 41(70 \%)$, compared to $105 / 204$ $52 \%$. For a $\mathrm{HCO} 3<22$ this was $20 / 23(87 \%)$ and $79 / 154(51 \%)$ respectively. If both were present, 10/12 (83\%) resulted in HFNC failure, compared to $80 / 143$ (56\%) without both present.

HFNC was started $19 \pm 15$ hours after extubation with no difference in the time between extubation and starting HFNC between the success and failure group ( $20 \pm 16$ hours vs. $18 \pm 15$ hours respectively, $p=0.35$ ). HFNC flow settings differed between the success and failure group ( $44 \pm 7 \mathrm{~L} / \mathrm{min}$ vs. $46 \pm$ $7 L /$ min, $p=0.007)$, but oxygen fraction was comparable ( $66 \pm 22 \%$ vs. $67 \pm 22 \% ; p=0.40)$.

Time spent on the HFNC differed between the groups; success group $44 \pm 47$ hours, failure group: $1 \pm 5$ hours, $p<0.001)$. After two hours treatment with HFNC the majority of patient with HFNC were already intubated, leaving 8 patients for further analysis. Breathing frequency was again a significant predictor for HFNC failure: mean $25 /$ min (SD 6) versus $21 /$ min (6) with HFNC success $(p<0.05)$. 
LOS in the ICU was for the failure group significantly longer than for the success group ( $405 \pm 519$ hours vs. $181 \pm 177$ hours $p<0.001$ ). ICU mortality was $7 \%$ in the success group vs. $15 \%$ in the failure group ( $p$ $=0.06)$ and hospital mortality $14 \%$ vs $24 \% \%(p=0.07)$. None of the subjects died in the 72 hours after the start of HFNC.

\section{Discussion}

The present study describes the differences in patients' characteristics between subjects that are being successfully treated with HFNC in the setting of post-extubation acute respiratory failure and subjects in which HFNC fails. HFNC was successful in $45 \%$ of the patients and could be stopped after a mean 44 hours. Independent predictors of HFNC failure were breathing frequency and blood gas $\mathrm{pH}$ at the start of HFNC. HFNC failure was associated with prolonged stay in ICU and there was a tendency to increased mortality.

To our knowledge our study is the largest study in literature documenting the use of HFNC for acute respiratory failure after extubation. There is one smaller study of Yoo et al., comparing HFNC and NIV therapy in 73 subjects with acute respiratory failure after extubation. ${ }^{11}$ They showed HFNC was noninferior to NIV in the avoidance of reintubation and associated with a shorter stay in the ICU. Reintubation could be avoided in $79 \%(n=27)$ of the patients treated with HFNC, which is a much higher rate compared to the reintubation avoidance rate of $45 \%$ in our cohort. In terms of age, sex and APACHE II score both cohorts are comparable. In their study the duration of mechanical ventilation prior to extubation was longer (132.7 \pm 85.6 hours) and the time between extubation and start of HFNC was shorter ( $8.6 \pm 11.8$ hours) compared to our cohort. Based on the data in the article of Yoo there is no clear explanation for the difference in reintubation avoidance rate between the two studies, except for the difference in methodological design.

In contrast to the scarcity of studies on the use of HFNC in the setting of post-extubation acute respiratory failure (ARF), there are numerous studies on the use of HFNC prior to (or the prevention of) intubation. Our success rate of $45 \%$ is comparable with the results of Rello who found a success rate of $45 \%(n=9)$ in patients treated with HFNC due to severe acute respiratory infection (2009 influenza $\mathrm{A} / \mathrm{H} 1 \mathrm{~N} 1)^{13}$, but less successful in comparison with Messika who found a HFNC success rates of $58 \%$ $(n=26)$ in the setting of ARDS. ${ }^{14}$

Nevertheless, not many studies are available exclusively focussing on the success or failure of HFNC in the setting of ARF. One of the largest randomized trials on HFNC compared the intubation rate of patients with acute hypoxemic respiratory failure treated their patient with either HFNC, conventional oxygen therapy (COT)or NIV and none of these three strategies showed any significant differences in intubation rate ( $38 \%$ vs. $47 \%$ vs. $50 \%$ respectively, $p=0.18$ for all comparisons). ${ }^{3}$

Primary outcome of our study showed a significant difference between the success and failure group for breathing frequency before the start of HFNC. After logistic regression breathing frequency remained an 
independent predictor of HFNC failure.

Previous, physical measurements on the use of HFNC revealed a significant reduction in median breathing frequency compared with non-rebreathing mask (NRM). ${ }^{15}$ This was confirmed by Sztrymf who not only found a significant reduction in breathing frequency $(p=0.009)$, but also a reduction in other respiratory parameters and heart rate. ${ }^{16}$ Additional analyses showed that 30 and 45 minutes after the start of $\mathrm{HFNC}$ a higher breathing frequency, a lower $\mathrm{SpO}_{2}, \mathrm{PaO}_{2}$ and $\mathrm{PaO}_{2} / \mathrm{FiO}_{2}$ ratio were associated with HFNC failure, with an increasing significance level over time. We also recorded respiratory characteristics at 2, 4, 8 and 24 hour after start of HFNC, but as majority of patients with HFNC failure were re-intubated within 2 hour after start, we could only do limited analysis on these data. From the results of Sztrymf we can conclude that HFNC failure can be expected when no improvement of respiratory parameters occurs after starting HFNC, and, combined with our data, HFNC failure is expected to occur within 2 hours after start of HFNC.

The difference in breathing frequency in our study (success: $22 \pm 7 / \mathrm{min}$ vs failure: $24 \pm 7 / \mathrm{min}$ ) is statistically significant, but its clinical applicability is more complicated.

The $\mathrm{pH}$ of the blood gas obtained (1 to 4 hours) prior to HFNC showed a tendency to significance and after logistic regression it came forward as a predictor for HFNC failure $(p=0.01)$. PaCOQ did not differ between the two groups. Post hoc analysis showed a higher risk of HFNC failure when $\mathrm{HCO}_{3}<22 \mathrm{mmol} / \mathrm{l}$. The differential diagnosis for a lowered $\mathrm{HCO}_{3}$ is broad. However in the setting of post-detubation failure there are some obvious causes such as a lowered circulating volume resulting in lactate acidosis, renal loss of bicarbonate in the setting of renal failure, or more theoretically, an excessive resuscitation with chloride. However we do not have any data on lactate or creatinine levels, diuresis or the amount of chloride that was used during resuscitation, so this differential diagnosis is pure speculative.

Secondary outcome of our study showed a difference in the LOS at the ICU after starting HFNC. No difference in ICU or in-hospital mortality was found between the groups. It is possible that this is due to a relative low sample size, although other authors with smaller simple sizes did find an effect of HFNC on mortality.

Messika did found a positive effect from HFNC success on mortality (ICU survival $96 \%$ vs. $50 \%$, $\mathrm{p}=0.01) .{ }^{14}$ In concordance, a significant difference in 90-day mortality was found for HFNC in acute respiratory failure when compared to COT or NIV. ${ }^{3}$ Although, this effect was not found in the study of Ni et al. when HFNC was started after extubation to prevent acute respiratory failure. ${ }^{4}$

There were several limitations to this study due to the retrospective design of the study, including lack of some data and indication for start of HFNC in some patients. In our centre the moment of extubation was not determined by a spontaneous breathing trial, but determined by the attending physician. As a result one can argue that the subjects in the failure group were extubated not under the right conditions. However, there was no difference between the two groups in the time between extubation and the start of HFNC. If patients were extubated under suboptimal conditions, one would expect them to be more 
dependent on respiratory support and as a result a smaller time frame between extubation and the start of HFNC, which is not the case.

Another limitation is that the arterial blood gasses were obtained 1 up to 4 hours before the start of HFNC. Therefore we do not have an accurate representation of the actual arterial blood gas at the start of HFNC and as a result no hard conclusions can be drawn regarding the higher risk of HFNC failure when a lower $\mathrm{pH}$ level is present. Also, due to the registration method we only have the SpO\&/FiOه ratio and not the more accurate $\mathrm{P} / \mathrm{F}$ ratio. At last, the study is hindered by all the limitations attached to its retrospective design. One of these limitations is that we cannot clarify on the considerations of the attending physician to start HFNC in the failure group, since apparently within a mean 1 hour (SD: 5 hours) it was decided that all these patients needed to be reintubated. You can wonder what the considerations were to still try HFNC in this group, what did the physician see in the clinical presentation of the patient which we cannot track down retrospectively in the available data?

With this study we aimed to identify patient characteristics that can predict failure of HFNC.

The need for predictors is evident. As found in a retrospective observational study of patients with respiratory failure in which the HFNC failed, a higher ICU mortality was present in patients intubated $>48$ hours after the start of HFNC (66.7\%) compared with patients intubated <48 hours $(39.2 \%, p=0.001) .{ }^{17}$ In our study we did also found a non-significantly higher mortality and a significant prolonged ICU-stay.

Roca et al focused in their study on early predictors of HFNC failure and developed a prediction tool that identifies the need for mechanical ventilation in patients treated with HFNC because of pneumonia induced hypoxemic acute respiratory failure. ${ }^{18}$ This so called ROX-index is defined as the ratio of pulse oximetry/fraction of inspired oxygen to breathing frequency. A recent subsequent study of the same author found the best prediction accuracy of the ROX index after 12 hours of HFNC treatment. ${ }^{19}$ However, due to the fact that subjects in which HFNC failed were quickly reintubated ( $1 \pm 5$ hours) we could not confirm this prediction accuracy after 12 hours in our study.

Clearly there is need for confirmation on the predictive value of patient characteristics and respiratory parameters on the failure of HFNC in prospective trials. Moreover, because the use of HFNC is widespread and the known adverse effects of HFNC failure are far-reaching.

\section{Conclusions}

In more than half of the patients HFNC failed when used for post-extubation failure. Breathing frequency and $\mathrm{pH}$ were predictors of HFNC failure. There was a non-significant higher ICU and in-hospital mortality in patients with HFNC failure; length of ICU stay was significant longer in the HFNC failure group.

\section{List Of Abbreviations}




$\begin{array}{ll}\text { APACHE IV } & \text { Acute Physiology And Chronic Health Evaluation } \\ \text { ARDS } & \text { acute respiratory distress syndrome } \\ \text { ARF } & \text { acute respiratory failure } \\ \text { CHF } & \text { congestive heart failure } \\ \text { COPD } & \text { Chronic Obstructive Pulmonary Disease } \\ \text { COT } & \text { conventional oxygen therapy } \\ \text { CPR } & \text { cardiopulmonary resuscitation } \\ \text { HFNC } & \text { High Flow Nasal Cannula } \\ \text { ICU } & \text { intensive Care Unit } \\ \text { LOS } & \text { length of stay } \\ \text { MDS } & \text { Minimal Data Set } \\ \text { NICE } & \text { National Intensive Care Evaluation } \\ \text { NRM } & \text { non-rebreathing mask } \\ \text { NIV } & \text { non-invasive ventilation } \\ \text { PDMS } & \text { Patient Data Management System }\end{array}$

\section{Declarations}

\section{Ethics approval and consent to participate}

The study was approved by the medical ethical committee of the Onze Lieve Vrouwe Gasthuis, Amsterdam. A waiver was given for informed consent due to the retrospective and non-invasive nature of this study. Reference number of this waiver: WO 14.112

\section{Consent for publication}

Not applicable

\section{Availability of data and materials}

The datasets used and/or analysed during the current study are available from the corresponding author on reasonable request

\section{Competing interests}

The authors declare that they have no competing interests

\section{Funding}

This study was conducted without any funding or financial support. No potential conflict of interest to declare. 


\section{Authors' contributions}

AT: literature search, analysis of data, manuscript preparation

LH: data collection, review of manuscript

ES: data collection, review of the manuscript

$A B$ : review of the manuscript

HE: literature search, data collection, study design, analysis of data, review of manuscript All authors have read and approved the manuscript.

\section{Acknowledgements}

Not applicable

\section{References}

1. Mauri T, Turrini C, Eronia N, et al. Physiologic Effects of High-Flow Nasal Cannula in Acute Hypoxemic Respiratory Failure. Am J Respir Crit Care Med. 2017;May 1(195 (9)):1207-1215.

2. Delorme M, Bouchard P-A, Simon M, Simard S, Lellouche F. Effects of High-Flow Nasal Cannula on the Work of Breathing in Patients Recovering From Acute Respiratory Failure. Crit Care Med. 2017:1.

3. Frat J-P, Thille AW, Mercat A, et al. High-Flow Oxygen through Nasal Cannula in Acute Hypoxemic Respiratory Failure. N Engl J Med. 2015;372(23):2185-2196.

4. Ni Y-N, Luo J, Yu H, et al. Can High-flow Nasal Cannula Reduce the Rate of Endotracheal Intubation in Adult Patients With Acute Respiratory Failure Compared With Conventional Oxygen Therapy and Noninvasive Positive Pressure Ventilation? Chest. 2017;151(4):764-775.

5. Thille AW, Boissier F, Ben-Ghezala $\mathrm{H}$, et al. Easily identified at-risk patients for extubation failure may benefit from noninvasive ventilation: A prospective before-after study. Crit Care. 2016;20(1):1-8.

6. Rochwerg B, Brochard L, Elliott MW, et al. Official ERS/ATS clinical practice guidelines: Noninvasive ventilation for acute respiratory failure. Eur Respir J. 2017;50(4).

7. Dhillon NK, Smith EJT, Ko A, et al. Extubation to high-flow nasal cannula in critically ill surgical patients. J Surg Res. 2017;217(310):258-264.

8. Hernández G, Vaquero C, Colinas L, et al. Effect of postextubation high-flownasal cannula vs noninvasive ventilation on reintubation and postextubation respiratory failure in high-risk patients a randomized clinical trial. JAMA - J Am Med Assoc. 2016;316(15):1565-1574.

9. Fernandez R, Subira C, Frutos-Vivar F, et al. High-flow nasal cannula to prevent postextubation respiratory failure in high-risk non-hypercapnic patients: a randomized multicenter trial. Ann Intensive Care. 2017;7(1).

10. Ni YN, Luo J, Yu H, et al. Can high-flow nasal cannula reduce the rate of reintubation in adult patients after extubation? A meta-analysis. BMC Pulm Med. 2017;17(1):1-10. 
11. Yoo JW, Synn A, Huh JW, Hong SB, Koh Y, Lim CM. Clinical efficacy of high-flow nasal cannula compared to noninvasive ventilation in patients with post-extubation respiratory failure. Korean $J$ Intern Med. 2016;31(1):82-88.

12. Zimmerman JE, Kramer AA, McNair DS, Malila FM. Acute Physiology and Chronic Health Evaluation (APACHE) IV: Hospital mortality assessment for today's critically ill patients. Crit Care Med. 2006;34(5):1297-1310.

13. Rello J, Pérez M, Roca 0 , et al. High-flow nasal therapy in adults with severe acute respiratory infection: a cohort study in patients with 2009 influenza A/H1N1v. J Crit Care. 2012;27(5):434-439.

14. Messika J, Ben Ahmed K, Gaudry S, et al. Use of High-Flow Nasal Cannula Oxygen Therapy in Subjects With ARDS: A 1-Year Observational Study. Respir Care. 2015;60(2):162-169.

15. Vargas F, Saint-Leger M, Boyer A, Bui NH, Hilbert G. Physiologic Effects of High-Flow Nasal Cannula Oxygen in Critical Care Subjects. Respir Care. 2015;60(10):1369-1376.

16. Sztrymf B, Messika J, Bertrand F, et al. Beneficial effects of humidified high flow nasal oxygen in critical care patients: A prospective pilot study. Intensive Care Med. 2011;37(11):1780-1786.

17. Kang BJ, Koh Y, Lim CM, et al. Failure of high-flow nasal cannula therapy may delay intubation and increase mortality. Intensive Care Med. 2015;41(4):623-632.

18. Roca O, Messika J, Caralt B, et al. Predicting success of high-flow nasal cannula in pneumonia patients with hypoxemic respiratory failure: The utility of the ROX index. J Crit Care. 2016;35(2016):200-205.

19. Roca O, Caralt B, Messika J, et al. An Index Combining Respiratory Rate and Oxygenation to Predict Outcome of Nasal High Flow Therapy. Am J Respir Crit Care Med. 2019;199(11):1368-1376. 